\title{
RA/DA cumulative curve analysis of local and diffuse neuroretinal rim area damage in glaucoma patients
}

\author{
Maurizio Rolando, Angelo Macrì, Michele Iester, Michele Altieri, Giovanni Calabria
}

\begin{abstract}
Aim-To evaluate the validity of cumulative rim/disc area (RA/DA) curve analysis as a clinical tool for the identification of glaucoma induced optic disc pathology. Methods-71 normal and 83 glaucomatous eyes were evaluated from a series of 154 subjects recruited for this study. For each eye, the cumulative distribution of RA/DA was calculated from 36 equally spaced rim sectors of each optic disc obtained by the automatic evaluation of simultaneous videographics (Image-net $X$ Rev.3/51b). To increase the sensitivity of this analysis in early glaucoma and in normal eyes, these cumulative curves were subsequently divided into two equal segments and the slopes of their respective regression lines compared.

Results-The median RA/DA value obtained from the 36 sectors was significantly different in glaucomatous eyes compared with normals ( $p<0.001)$. Nevertheless, the curves (5th-95th percentile of the cumulative curves distribution) of early glaucomatous eyes fell within the normal range. When the cumulative curve of these marginal cases was then divided into two equal segments, the comparison of the slopes of the regression lines showed a significant difference ( $p$ $<0.05$ ) in $100 \%$ of early glaucomatous eyes. Furthermore, normal eyes were shown to be true negatives in $93 \%$ of the cases in which no significant difference between the two slopes was observed.

Conclusion-Analysis of the RA/DA cumulative curve from 36 sectors of the optic disc was a valid method for the identification of glaucomatous disc pathology; however, a further calculation of the slopes of the two RA/DA regression lines was needed to identify early glaucomatous damage.
\end{abstract}

University Eye Clinic, Genoa, Italy

M Rolando

A Macrì

M Iester

M Altieri

G Calabria

Correspondence to:

Angelo Macrì, MD, Clinica

Oculistica dell'Università $\mathrm{d}$ Genova, Largo R

Bensi-Ospedale S Martino

Pad 9, 16132 Genoa, Italy.

Accepted for publication 29 January 1998 been introduced for the study of the morphology and morphometry of the optic disc. From this, much quantitative information is now readily available on optic disc variables in normal and pathological conditions; however, the clinical relevance of these data is not always evident. A properly selected statistical elaboration of the raw data accumulated by these potentially useful systems might render their numerical results more significant, thus allow- ing for a clinical interpretation or definition of these variables, as has happened with computerised perimetry. ${ }^{1-3}$

A quantitative analysis of the cumulative defect curve (Bebie curve) to rim size measurements might allow for the identification of rim area size changes, resolving the difficulty of detection of optic disc defects and the distinction between global and localised damage for the diagnosis and follow up of glaucomatous changes. ${ }^{4}$

The aim of the present study was the development and testing of a new method, obtained by modifying the Bebie cumulative defect curve, to be used as a clinical tool to distinguish normal from glaucomatous optic discs and, in glaucoma, to separate diffuse from localised neuroretinal rim area damage. ${ }^{56}$

\section{Materials and methods}

SUBJECTS

One hundred fifty four subjects were recruited from January 1995 to September 1996. Glaucomatous patients were consecutively selected from the glaucoma service of the department of ophthalmology of the University of Genoa, while the normals were volunteers. One eye from each subject was randomly chosen.

Exclusion criteria were the following:

- refractive defects $>$ plus or minus 3 dioptres (spherical equivalent)

- inability to obtain a mydriasis $>7 \mathrm{~mm}$

- the presence of systemic and/or ocular diseases able to modify papillary surface (diabetes, hypertension, vasculitis, other primary and/or secondary vascular diseases, optic neuropathy except glaucoma, etc)

- dioptric media opacities able to hinder good image acquisition

- inability to obtain a reliable (less than $30 \%$ false positive and false negative responses and fixation loss less than 10) 30-2 Humphrey visual field (Humphrey VFA 640, program "central 30-2") within 15 days of optic disc image acquisition.

The inclusion criteria for glaucoma subjects In recent years, computerised systems have were:
- the presence at two or more visits of an intraocular pressure higher than $21 \mathrm{~mm} \mathrm{Hg}$ without therapy

- the presence of (a) typical perimetric glaucomatous defects identified from a reliable 10 $\mathrm{dB}$ loss or greater in the superior or inferior Bjerrum areas when compared with perimeter defined age matched controls; (b) three contiguous points with $5 \mathrm{~dB}$ loss or greater in the superior or inferior Bjerrum 
Right eye

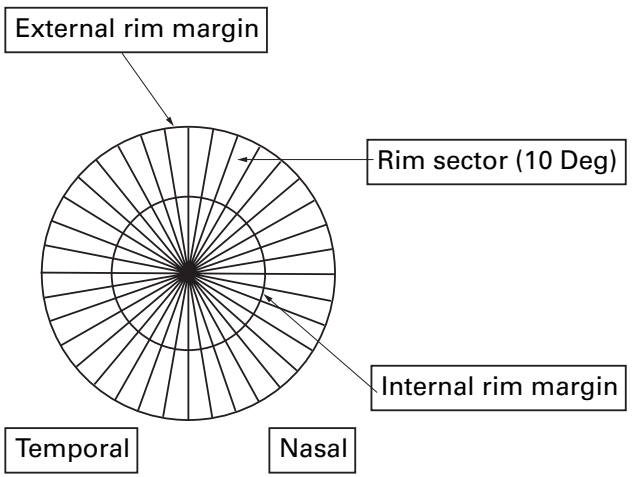

Figure 1 Cumulative $R A / D A$ curves were generated in normal and glaucomatous patients by first dividing the rim of the optic disc into 36 equally spaced sectors, then sorting decrementally the rim/disc area of each sector to form a cumulative curve.

Table 1 Demographics (mean (SEM)) and optic disc morphological characteristics of normal and glaucomatous patients

\begin{tabular}{llll}
\hline Variable & $\begin{array}{l}\text { Normals } \\
(n=71)\end{array}$ & $\begin{array}{l}\text { Glaucoma } \\
(n=83)\end{array}$ & $\begin{array}{l}\text { p Value } \\
\text { (Mann-Whitney) }\end{array}$ \\
\hline Male/female & $39 / 32$ & $35 / 48$ & - \\
Age (years) & $55(12.5)$ & $57.1(14.2)$ & Not significant \\
Disc area $\left(\mathrm{mm}^{2}\right)$ & $2.40(0.27)$ & $2.43(0.24)$ & $\begin{array}{l}\text { Not significant } \\
\text { Rim/disc area }\left(\mathrm{mm}^{2}\right)\end{array}$ \\
Mean deviation $(\mathrm{dB})$ & $0.61(0.05)$ & $0.47(0.04)$ & $<0.001$ \\
Corrected pattern standard deviation $(\mathrm{dB})$ & $-0.93(0.79)$ & $-5.53(6.96)$ & $<0.001$ \\
& $0.4(0.6)$ & $5.9(2.45)$ & $<0.001$ \\
\hline
\end{tabular}

areas; or (c) a $10 \mathrm{~dB}$ difference across the nasal horizontal midline in two or more adjacent locations. No point was on the edge except immediately above or below the nasal horizontal meridian ${ }^{78}$

- optic disc appearance was not considered a criterion for inclusion into the study.

Inclusion criteria for normal subjects were the following:

- intraocular pressure lower than $21 \mathrm{~mm} \mathrm{Hg}$ on at least two measurements

- no personal or familial history of high intraocular pressure

- absence of any kind of perimetric defect.

IMAGE ACQUISITION

Image acquisition was performed by means of the Image-net X Rev-3.51b Topcon system (Topcon Europe, Netherlands) of optic disc simultaneous stereoscopic videographics. This

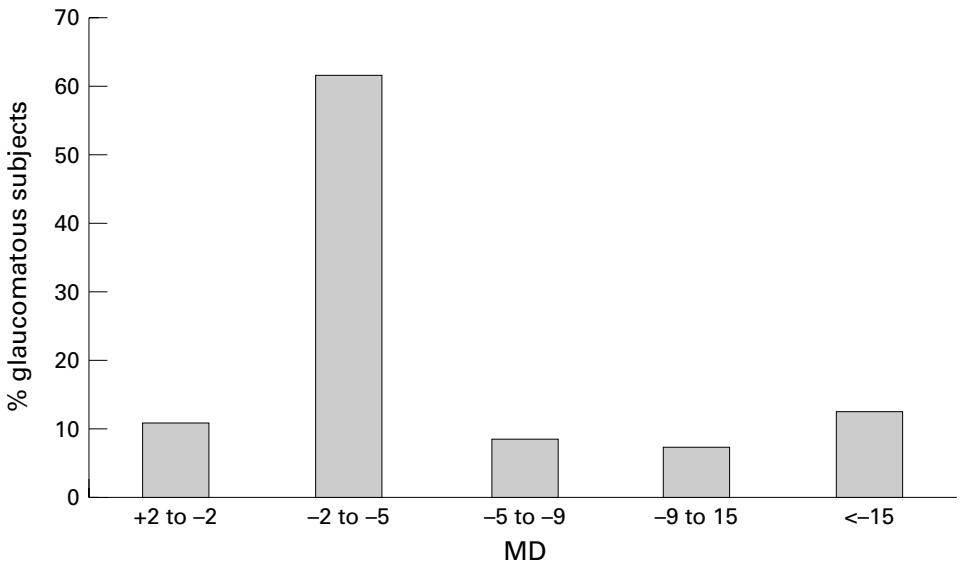

Figure 2 Distribution (percentage) of glaucomatous patients according to mean deviation (MD). system includes two charge coupled device video cameras with a field of view of 12 degrees and a green separation system to further delineate the topography of the optic disc. Analogue signals coming from the two cameras travel to the red and green analogue to digital converters, with the resulting digital images $(512 \times$ $512 \times 24$ bit image memory) displayed to the operator and then saved to the disk. ${ }^{9}$ Correction for magnification errors is provided by means of Littman formula based correction factors implemented in the software. ${ }^{9}$

For each eye, at least three stereoscopic images of the optic nerve were taken, and the mean and standard deviation from the mean of the three $\mathrm{RA} / \mathrm{DA}$ values were calculated. The value of the coefficient of variation (standard deviation/average $\times 100$ ) was determined for each eye. When the coefficient of variation was higher than $7 \%$, the images were discarded and a new image acquisition was performed.

The operator identified four points located on the outer edge of the optic disc rim on each optic disc image using the program "Optic Disc Analysis". This program, which defines the external edge of excavation as the point 150 $\mu \mathrm{m}$ under the plane in contact with the disc borders, allows the calculation of the traditional bidimensional morphological variables: rim area (RA), disc area (DA), rim/disc area ratio (RA/DA ratio), cup volume (CV), cup/disc ratio vertical $(\mathrm{C} / \mathrm{D} \mathrm{V})$, horizontal (C/D H), and C/D. ${ }^{9}$

The RA/DA ratio was then calculated for each patient in order to evaluate the cumulative distribution of the areas of the 36 equally spaced (10 degrees) sectors of the optic disc (Fig 1). For every sector, the data for normal and glaucomatous optic discs were separately and decrementally sorted to form a line.

The 50th (median), 95th, and 5th percentile were calculated for each sector and the values were graphically presented allowing a rapid visualisation of data from normal and pathological eyes. The data distribution was not "normal" and thus the non-parametric MannWhitney test was used to compare the difference between the medians of the 36 RA/DA sectors of glaucomatous and normal eyes.

For all curves obtained from normal eyes and for those curves from glaucomatous eyes which fell within the normal range, the curve was divided into two equal parts with the same

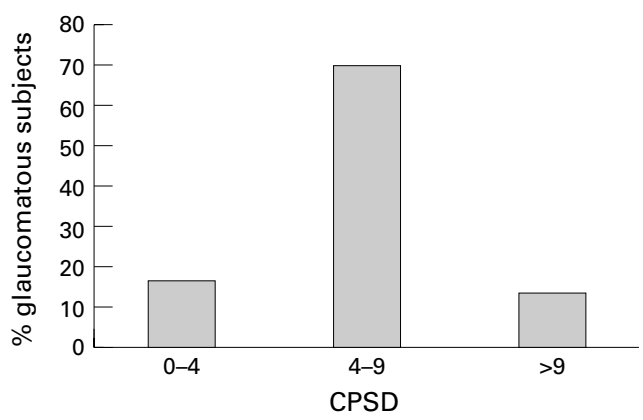

Figure 3 Distribution (percentage) of glaucomatous patients according to the corrected pattern standard deviation (CPSD). 


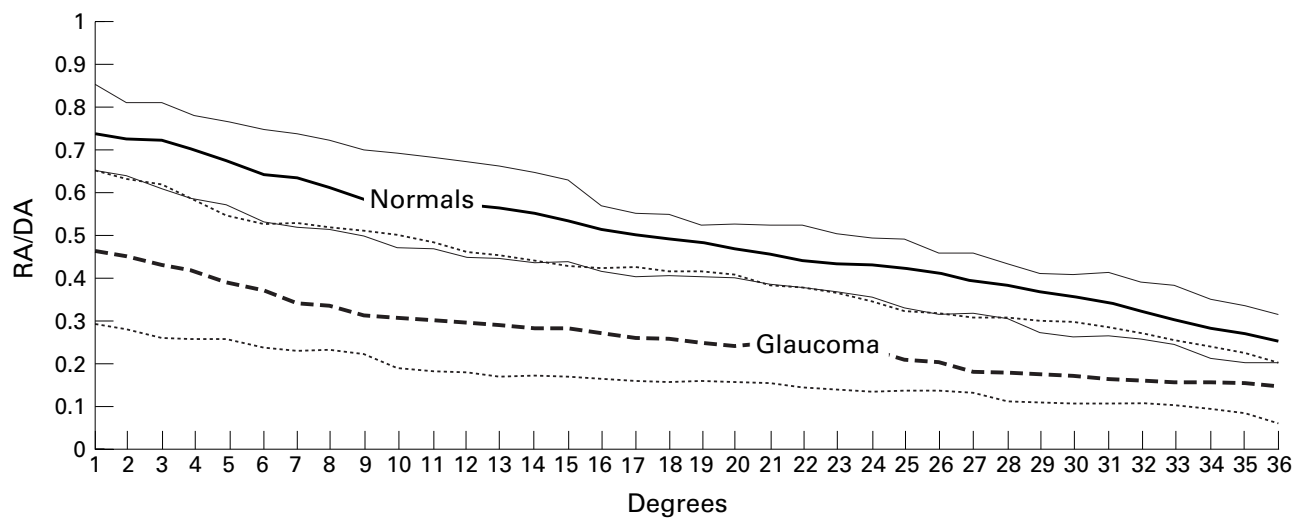

Figure 4 Cumulative curve distribution of the rim/disc area in normal (continuous lines) and glaucomatous (broken lines) eyes. Evaluation of the median and the distribution of the 5th and 95th percentile allowed for a rapid visualisation of normal and glaucomatous discs (dotted lines: 5th and 95th percentile of glaucomatous eyes; broken line: median of glaucomatous eyes; thin continuous lines: 5 th and 95 th percentile of normal eyes; thick continuous line: median of normal eyes).

Table 2 Sensitivity and specificity of $R A / D A$ cumulative distribution curves in distinguishing normal from glaucomatous eyes. An increase in sensitivity was demonstrated after further analysis of the cumulative curves by separating them into two equal parts and comparing their respective slopes

\begin{tabular}{lll}
\hline & $\begin{array}{l}\text { RA/DA cumulative } \\
\text { curves }\end{array}$ & $\begin{array}{l}\text { After slope } \\
\text { comparison }\end{array}$ \\
\hline Sensitivity & $92.8 \%$ & $100 \%$ \\
Specificity & $90.1 \%$ & $90.1 \%$ \\
\hline
\end{tabular}

number of points $(n=18)$ and the regressions of these partial curves were calculated. The RA/DA cumulative curve was then determined by comparing the slopes of the regression lines of the two partial curves.

The EXCEL 5.0 (Microsoft) software program was used for graphic presentation of the data and the GraphPad Prism software (Version 2.0) was used for statistical analyses, including the comparison of the slopes of the regression lines.

\section{Results}

The group of subjects who matched the inclusion and exclusion criteria was composed of 71 normal and 83 glaucoma patients. Age, sex,

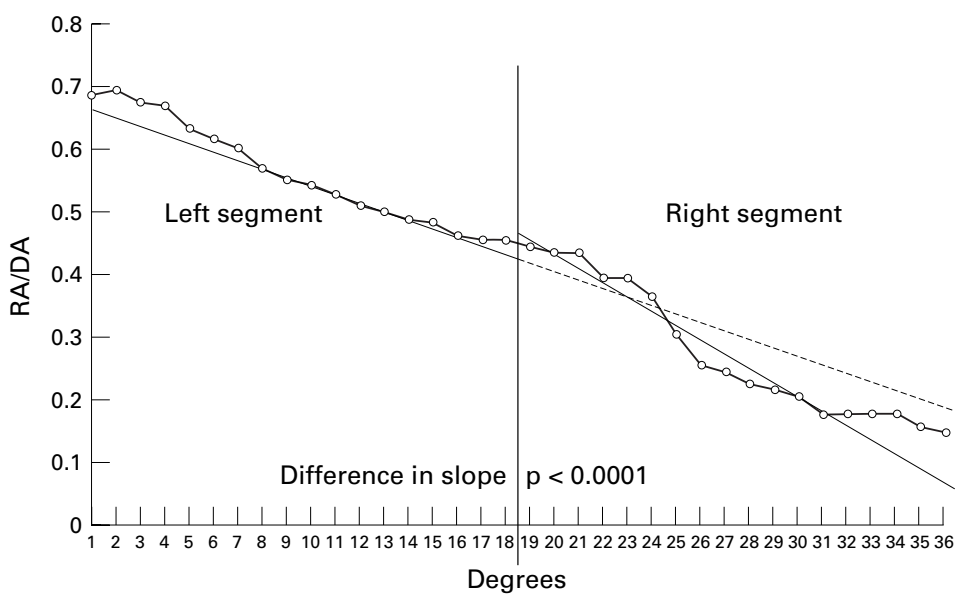

Figure 5 Cumulative curve of one of the six optic discs in which there was an overlapping of the 5th-95th percentile distribution of glaucomatous and normal eyes - that is, a glaucomatous curve within the normal range. The cumulative curve was then divided into two equal segments and the difference between the slope of the regression lines calculated. perimetric and morphometric characteristics of the participants are detailed in Table 1. In the glaucomatous group, the distributions of the deviation from the mean (MD) and the corrected pattern standard deviation were analysed, the details of which are illustrated in Figures 2 and 3. The difference between the medians of the $36 \mathrm{RA} / \mathrm{DA}$ sectors of glaucomatous and normal eyes was highly statistically significant using the Mann-Whitney test ( $p$ $<0.001$ ). Figure 4 illustrates the difference in position on a Cartesian diagram of the median, 5 th, and 95th percentile of focal (10 degrees) RA/DA cumulative curves of normal and glaucomatous eyes.

Sensitivity and specificity of RA/DA cumulative curves in distinguishing normal from glaucomatous optic discs were then calculated. True positives were defined as glaucomatous eyes with curves which fell outside the range obtained from normal eyes. True negatives were defined as normal eyes with curves within the normal range. Table 2 describes these results.

Using the above definitions, six eyes of the glaucomatous group were identified as false negatives - that is, the RA/DA cumulative curve fell within the cumulative curve distribution of normal eyes. However, in all of these eyes, when the curve was divided into two equal parts, the slopes of the two regression lines were significantly different (at least $\mathrm{p}$ $<0.05)$. This further data manipulation also demonstrated $93 \%$ of normal eyes to be true negatives - that is, to have cumulative curves within the normal range and no statistically significant change between the slopes of the two partial curves (Fig 5).

\section{Discussion}

Computerised analysis of the optic disc provides a wealth of quantitative data as to its morphology both in normal and glaucomatous eyes. A proper statistical management of these data is needed to render the information clinically relevant and utilisable in the diagnosis and assessment of optic disc changes in glaucoma. ${ }^{10-12} \mathrm{~A}$ similar evolution in clinical practices occurred in 1987, when Flammer 
suggested the formulation of perimetric indices for the interpretation of raw data provided by computerised perimetry in order to obtain useful clinical information.

Rim area measurements taken by computerised optic disc analysers are reported to be one of the best indicators of glaucoma induced optic disc changes. ${ }^{2} 31314$ However, when a localised defect which reduces the size of the rim area is present, the machine can not distinguish this localised defect from a diffuse rim fibre loss. This difficulty in separating localised from global defects, which was also a shortcoming of visual field analysis, was brilliantly solved by the introduction of the cumulative defect curve (Bebie curve). ${ }^{4}$

In 1996, both Bartz-Schmidt and Asawaphureekorn demonstrated in a small number of normal and glaucomatous patients that a statistical elaboration of cumulative defect curve data resulted in a rapid and easy method to identify the presence of diffuse and localised defect areas of the disc rim. ${ }^{5}$ Applying this method to the presently studied, larger group of glaucomatous patients in different stages of the disease yielded very favourable results, with a sensitivity of $92.8 \%$ and a specificity of $90.1 \%$ in distinguishing normal from glaucomatous eyes.

No disc area of our sampling was outside the mean disc size range, so there was no need to use the above method for evaluation. The outsiders in disc size could distort RA/DA ratio. ${ }^{15}{ }^{16}$ Small optic discs may have an RA/DA curve within the normal range despite acquired tissue loss because of a falsely high RA/DA ratio. Conversely, large discs may have an RA/DA ratio outside the normal range without pathological nerve fibre loss. In these extreme cases of disc size, the Bartz-Schmidt correction factor may be applied. ${ }^{5}$ This was evident in early glaucoma patients, probably because of greater size variability of the rim area, resulting in cumulative RA/DA curves which were sometimes partially or totally included within the range of normal subjects, giving rise to a small number of false negatives. Thus, in marginal cases, evaluation of the cumulative RA/DA curve, alone, was not sufficient for an accurate identification of disc pathology.

In an attempt to solve this problem, the cumulative curves of these early glaucomatous patients were divided into two equal segments and the slopes of their regression lines were compared. Results demonstrated that in glaucomatous eyes with the RA/DA cumulative curve located above the 5 th percentile of normal eyes, there was a statistically significant difference between the slopes of the two regression lines. This different inclination between the slopes of the regression lines of the two partial arcs indicated the presence of a localised thinning of the optic disc rim. Applying this corrective data elaboration to the data from the six false negatives subjects improved test sensitivity from $92.8 \%$ to $100 \%$.

The group of glaucomatous patients enrolled in the study included mainly patients with initial but definite glaucomatous visual field changes (Figs 2 and 3 ).
Glaucoma was diagnosed in this study only by the presence of typical visual field changes, and not by any morphological or morphometric changes of the optic discs in order to assure a heterogenous sampling of glaucoma patients and potential disc pathologies. However, these inclusion criteria limited the entry of subjects with definite optic disc changes yet no visual field changes and those with field defects which cannot be identified as glaucomatous. ${ }^{8}$ For example, patients with uniform loss in differential light sensitivity or patients characterised by scattered field damage were not entered and, thus, the potential of the RA/DA cumulative curve test to identify early disc pathology in this population was not investigated. ${ }^{17} 18$

In conclusion, while other diagnostic tests are needed to identify precisely the site of optic disc damage, the cumulative RA/DA curve method was shown to be useful in the detection of glaucoma induced disc pathology and in the recognition of diffuse versus local damage. In marginal cases of early glaucoma in which the sensitivity of this method was limited, a subsequent analysis, dividing the cumulative curve into two equal parts and comparing the slopes of their respective regression lines, improved test sensitivity to $100 \%$, allowing for identification of even early optic disc damage in these patients.

1 Flammer J, Jenni F, Bebie JH, et al. The Octopus glaucoma G-1 program. Glaucoma 1987; 9:67-72.

2 Caprioli J, Miller JM. Videographic measurements of optic nerve topography in glaucoma. Invest Ophthalmol Vis Sci 1988;29:1294-8.

3 Damms T, Dannheim F. Sensitivity and specificity of optic disc parameters in chronic glaucoma. Invest Ophthalmol Vis Sci 1993;34:2246-50.

4 Bebie H, Flammer J, Bebie Th. The cumulative defect curve: separation of local and diffuse components of visual field damage. Graefes Arch Clin Exp Ophthalmol 1989;227: $9-12$.

5 Bartz-Schmidt KU, Sengersdorf A, Esser P, et al. The cumulative normalized rim/disc area ratio curve. Graefes Arch Clin Exp Ophthalmol 1996;234:227-31.

6 Asawaphureekorn S, Zangwill L, Weinreb RN. Rankedsegment distribution curve for interpretation of optic nerve topography. F Glaucoma 1996;5:79-90.

7 Caprioli J. The contour of the juxtapapillary nerve fiber layer in glaucoma. Ophthalmology 1990;97:358-65.

8 Caprioli J. Clinical evaluation of the optic nerve in glaucoma. Trans Am Ophthalmol Soc 1994;92:589-641.

9 Varma R. The Topcon image analyzer. In: Varma R, Spaeth GL, Parker KW, eds. The optic nerve in glaucoma. Philadelphia: JB Lippincott, 1993:255-68.

10 Iester M, Traverso CE, Rolando M, et al. Study of the correlation between average values of optic disc parameters and their measurement variability using stereovideographic digital analysis. Ophthalmologica 1995;209:177-81.

11 Rolando $M$, Iester $M$, Campagna $P$, et al. Measurement variability in digital analysis of optic discs. Doc Ophthalmol 1994;85:211-22.

12 Rolando M, Macrì A, Altieri M, et al. Morphometric analysis of the optic disc surface: the level of smoothness as a diagnostic parameter for glaucoma. Int Ophthalmol 1997; 20:15-20.

13 Iester M, Mikelberg FS, Swindale NV, et al. ROC analysis of Heidelberg retina tomograph optic disc shape measures in glaucoma. Can f Ophthalmol 1997;32:382-8.

14 Iester M, Mikelberg FS, Courtright P, et al. Correlation between the visual field indices and Heidelberg retina tomograph parameters. F Glaucoma 1997;6:78-82.

15 Gusek GC, Jonas JB, Naumann GOH. Is there a difference in optic disc size between normal and glaucomatous eyes? Fortschr Ophthalmol 1988;85:52-3.

16 Iester M, Mikelberg FS, Drance SM. The effect of optic disc size on diagnostic precision with the Heidelberg retina tomograph. Ophthalmology 1997;104:545-8.

17 Anctil JL, Anderson DR. Early foveal involvement and generalized depression of the visual field in glaucoma. Arch Ophthalmol 1984;102:363-70.

18 Caprioli J, Sears M, Miller JM. Patterns of early visual field loss in open-angle glaucoma. Am f Ophthalmol 1987;103: $512-17$. 